\title{
Studies on the growth and characterization of L-cysteine hydrogen fluoride single crystal
}

\author{
AzeezaA Varsha Mohammed ${ }^{1}$, Suresh Sagadevan ${ }^{2, *}$ \\ ${ }^{1}$ Department of Physics, JEPPIAAR SRR Engineering College, Chennai-603 103, India \\ ${ }^{2}$ Department of Physics, AMET University, Chennai-603 112, India
}

\begin{abstract}
L-cysteine hydrogen fluoride (LCHF) single crystals were grown from aqueous solution. Single crystal X-ray diffraction, FT-IR, UV-Vis-NIR, and TG-DTA were used to test the grown crystals. The specimen dielectric and mechanical behaviors were also studied. Powder X-ray diffraction of the grown crystal was recorded and indexed. The optical properties of the LCHF crystal were determined using UV-Vis spectroscopy. It was found that the optical band gap of LCHF was $4.8 \mathrm{eV}$. The crystal functional groups were identified using FT-IR. Second harmonic generation (SHG) efficiency of the LCHF was three times higher than that of KDP. The dielectric constant, dielectric loss and AC conductivity were measured at different frequencies and temperatures.
\end{abstract}

Keywords: nonlinear optical material; solution growth; optical transmission; second harmonic generation (SHG), TG-DTA, DSC, dielectric

\section{Introduction}

Over the past few decades, new nonlinear optical (NLO) materials, structures, and devices have been developed to drive nonlinear optics from the laboratory to real applications. The materials have a wide range of applications including communications, optical storage and optical computing. For a wide variety of processes, the rapidly developing field of NLO research and applications still needs new materials. Nowadays, KDP is the most widely used NLO crystal for Nd:YAG laser SHG. However, due to the changes caused by refractive, optical and hygroscopic damage, there are many problems associated with the use of KDP crystals. Research into new NLO crystals is, therefore, important [1-3]. Considerable theoretical and experimental investigations were carried out to understand the microscopic origin of the nonlinear behavior of organic NLO materials. NLO frequency conversion material generally consists of an electron donor, an acceptor, and a conjugated $\pi$-system as a bridge providing electronic communication between the donor and the recipient $[4,5]$. The benefits of organic over inorganic systems include high

*E-mail: sureshsagadevan@gmail.com electronic sensitivity resulting from high molecular polarization, fast response time, easy modification through standard synthesis methods and relative ease of device processing. Amino acids and their complexes are a part of family of organic materials considered for photonic applications. Photonic crystals that prohibit the propagation of light within a band gap for some frequencies enabled exciting new ways of controlling and building integrated optical devices. L-cysteine is an organic compound in the category of amino acids. It is one of the amino acids that occur naturally $[6,7]$. L-cysteine exists in zwitterionic form and is considered as a potential candidate for piezoelectric and non-linear applications in a noncentrosymmetric space group. L-cysteine in a solid-state exists as dipolar ion in which carboxyl group is present as a carboxylate ion and amino group as ammonium ion. Furthermore, a thiol group is present in the L-cysteine aqueous solution $[8,9]$.

\section{Material synthesis}

The title compound LCHF was grown using the technique of slow solvent solution evaporation. Commercially available AR grade L-cysteine 
and hydrofluoric acid in the stoichiometric ratio 1:1 were used as starting materials for synthesizing the title compound. Appropriate quantities of these materials were dissolved in deionized water and the solution was filtered through Whatman filter paper. Then, the filtered solution was kept in a dustless environment and optimally closed for controlled evaporation. Successive recrystallization process enhanced further the purity of the synthesized salt. In a period of 32 days, a transparent single crystal with a size of $18 \mathrm{~mm} \times 13 \mathrm{~mm} \times 11 \mathrm{~mm}$ was harvested. The grown single crystals of LCHF are shown in Fig. 1.

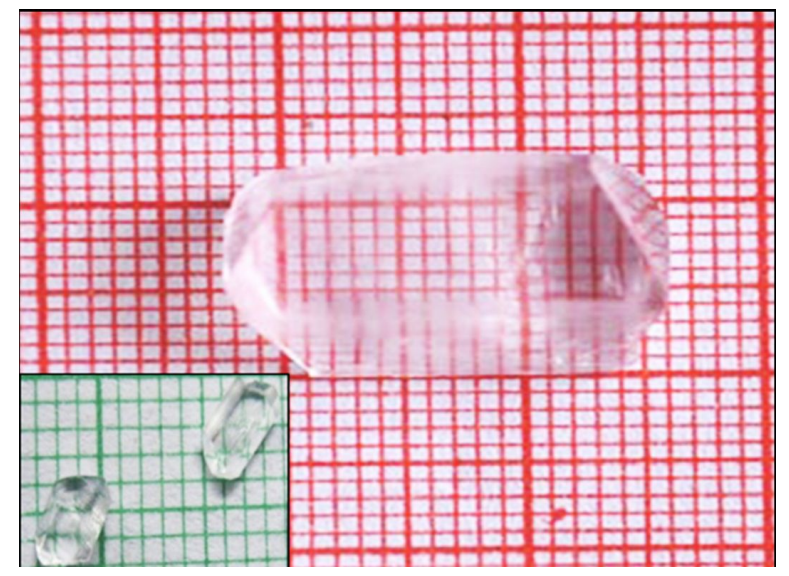

Fig. 1. The grown LCHF single crystal.

\section{Results and discussion}

\subsection{Single crystal $X$-ray diffraction}

Single crystal X-ray diffraction study was performed to identify the structure of the grown LCHF crystals using Bruker APEX II CCD area detector diffractometer equipped with graphite monochromatized MoK $\alpha$ radiation with a wavelength of $\lambda=0.7103 \AA$. The obtained lattice parameters were $\mathrm{a}=11.78 \AA, \mathrm{b}=5.32 \AA$ and $\mathrm{c}=15.43 \AA$, $\alpha=\gamma=90^{\circ}, \beta=106.7^{\circ}$, unit cell volume $=913 \AA^{3}$. It was confirmed by XRD analysis that the grown crystal had a monoclinic crystal structure with the space group $\mathrm{P}_{21}$, which was in good agreement with reported data [10]. It was also confirmed that LCHF was not incorporated in the organic host lattice sites. Fig. 2 shows the atomic numbering scheme of the grown crystal.

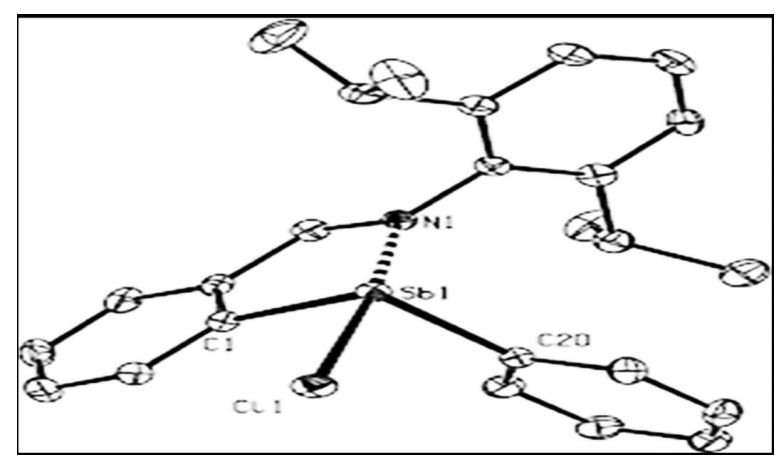

Fig. 2. ORTEP scheme of LCHF.

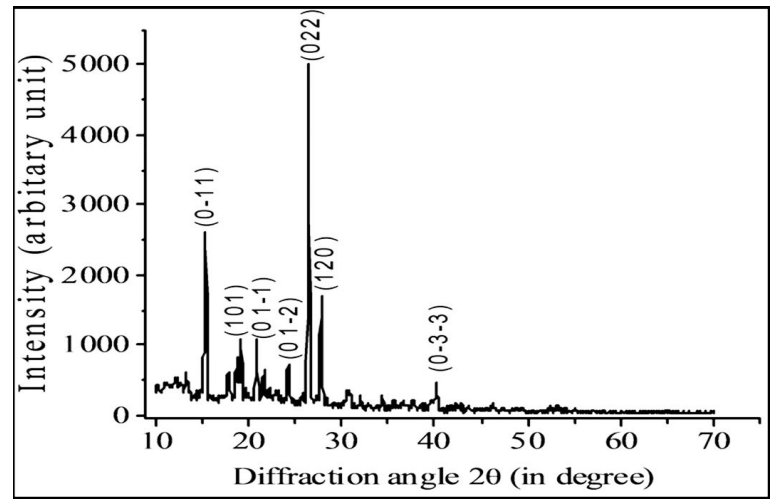

Fig. 3. Powder XRD pattern of LCHF.

\subsection{Powder X-ray diffraction}

A powdered sample was subjected to X-ray diffraction study using a diffractometer SEIFERT, JSO- DEBYEFLEX 2002 (GERMANY) with characteristic $\mathrm{CuK} \alpha$ radiation in the range of $10^{\circ}$ to $70^{\circ}$ at a scan rate of $2 \% \mathrm{~min}$. The grown crystal powder XRD profile is shown in Fig. 3. The sharp and strong peak appearance confirms good crystalline nature of the grown sample. The estimated lattice parameters of the crystal agree well with the reported data. The slight shift in the sharp peak position at $27.82^{\circ}$ may be due to the addition of hydrogen fluoride to the crystal and is also confirmed by the slight variation in the lattice parameters of the grown crystal. 


\subsection{FT-IR analysis}

The FT-IR spectrum of the grown crystal in the spectral range of $4000 \mathrm{~cm}^{-1}$ to $400 \mathrm{~cm}^{-1}$ was recorded by BRUKER IFS 66V infrared spectrophotometer. Fig. 4. shows the resulting FTIR spectrum. The wide band around $3450 \mathrm{~cm}^{-1}$ is due to the presence of $\mathrm{OH}$. The peaks at frequencies of $3003 \mathrm{~cm}^{-1}$ and $2293 \mathrm{~cm}^{-1}$ are attributed to $\mathrm{NH}_{3}^{+}$stretching and $\mathrm{C}-\mathrm{H}$ stretching, respectively. $\mathrm{N}-\mathrm{H}$ bending vibration is assigned to the peak at $1505 \mathrm{~cm}^{-1}$. The peaks at $1171 \mathrm{~cm}^{-1}$ and $1048 \mathrm{~cm}^{-1}$ reveal the presence of $\mathrm{C}-\mathrm{N}$ group. The stretching bands $\mathrm{S}-$ $\mathrm{H}$ and $\mathrm{C}-\mathrm{S}$ appear at $2515 \mathrm{~cm}^{-1}$. The group of $\mathrm{S}-\mathrm{H}$ thiol is observed at $2571 \mathrm{~cm}^{-1}$ [11]. The band of $\mathrm{C}-\mathrm{S}$ absorption is $664 \mathrm{~cm}^{-1}$. $\mathrm{N}-\mathrm{H}$ bending vibration is assigned to the peak at $1505 \mathrm{~cm}^{-1}$. The sharp, strong band at $1723 \mathrm{~cm}^{-1}$ shows the $\mathrm{C}=\mathrm{O}$ stretching of $\mathrm{COOH}$ group. The peaks observed at $816 \mathrm{~cm}^{-1}$ and $533 \mathrm{~cm}^{-1}$ indicate the presence of $\mathrm{CH}_{2}$ group while the $\mathrm{COO}$ group is attributed to the peak observed at $1586 \mathrm{~cm}^{-1}$. It is also confirmed from the above discussion that the amino acid cysteine containing sulfur is in its zwitterionic state.

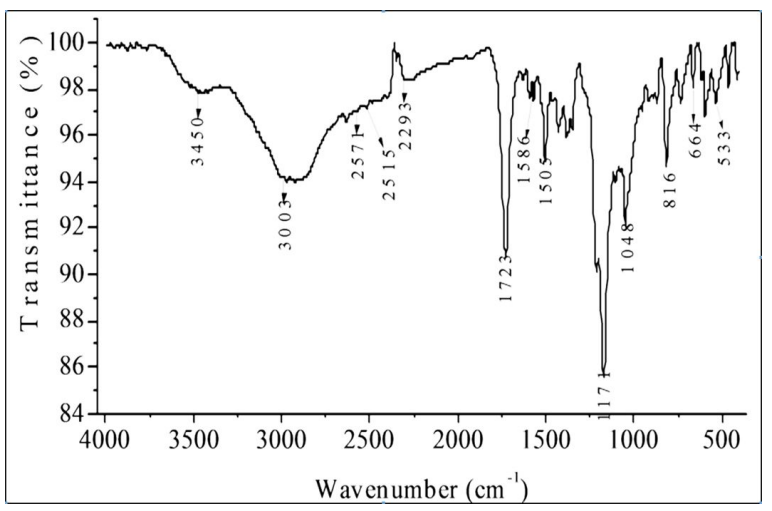

Fig. 4. FT-IR spectrum of LCHF.

\subsection{UV-Vis-NIR studies}

The optical transparency of the grown crystal was studied by the UV-Vis-NIR. The optical absorption spectrum was recorded using UV-Vis-NIR spectrometer in the wavelength range of $200 \mathrm{~nm}$ to $1200 \mathrm{~nm}$. The obtained transmission spectrum is shown in Fig. 5 in which the absorption edge is observed at $228 \mathrm{~nm}$ in the lower wavelength region. It is noticed that the UV spectrum shows the presence of a wide transparency window lying between $320 \mathrm{~nm}$ and $1200 \mathrm{~nm}$ at $\lambda_{\max }=228 \mathrm{~nm}$. The prohibited energy gap was estimated as $5.45 \mathrm{eV}$, which is a typical value of any dielectric material [12], from the relationship $E_{g}=1.243 \times 10^{3} / \lambda_{\max }$. It is, therefore, evident from the analysis of the optical transmission spectrum that the grown crystal is transparent in the visible region without any absorption peak, which is the essential requirement for any nonlinear optical crystal used in parametric oscillator, SHG and similar applications.



Fig. 5. Transmission spectrum of LCHF.

\subsection{Second harmonic generation test}

Kurtz and Perry powder technique [13] confirmed the relative second harmonic generation activity of the grown crystal. A finely ground powdered crystal was taken in a capillary tube for this study. The Q-switched Nd:YAG laser beam with $10 \mathrm{~ns}$ pulse width and $10 \mathrm{~Hz}$ repetition rate was used to test the sample NLO properties. The second harmonic signal generated in the sample was confirmed by green crystal radiation emission. The crystal can, therefore, be used for photonic and optoelectronic device applications.

\subsection{TG-DTA analysis}

The LCHF crystal TG/DTA curve is shown in Fig. 6. The DTA curve shows a $253{ }^{\circ} \mathrm{C}$ endothermic peak which corresponds to decomposition 
temperature of LCHF. L-cysteine decomposition temperature is $240{ }^{\circ} \mathrm{C}$. This higher decomposition temperature results probably from reinforcing the lattice with hydrogen fluoride present inside the crystal. Due to the addition of hydrogen fluoride, the decomposition temperature of LCHF increased by $13{ }^{\circ} \mathrm{C}$.



Fig. 6. TG-DTA curve of LCHF.

\subsection{Dielectric analysis}

The variations in the dielectric constant and dielectric loss with frequency at different temperatures are shown in Fig. 7 and Fig. 8, respectively. Fig. 7 shows the plot of dielectric constant versus $\log$ frequency. It is found that the dielectric constant is high at low frequencies and decreases with increasing frequency. This may be mainly due to the loosely bonded ions in the crystal at lower frequency region. Significant conductance is observed at low frequency region. In both cases, the values are found to rise as temperature increases and decrease as frequency increases. This is a normal dielectric behavior $[14,15]$. It means that the mechanism of polarization is related to the process of conduction. The electronic exchange of ions in the crystal provides local electrons displacement in the direction of the applied field, which in turn, results in polarization. The present study, therefore, indicates that LCHF is not only a potential NLO material but also its low dielectric constant value that may be useful for microelectronic industries and modulators of electro-optics. Crystal expansion, electronic and ionic polarizations and the presence of impurities and crystal defects are generally responsible for the variation of dielectric constant with temperature. The increase at higher temperatures can be attributed to the heat-generated carriers and impurity dipoles [16]. No significant volume change is expected in the studied temperature range. Thus, the observed increase in both dielectric constant and dielectric loss may be due to thermal expansion when the temperature is increased. Similarly the dependence of the dielectric loss with frequency is shown in Fig. 8. The high dielectric loss at low frequency is found to decrease in the higher frequency region. The low value of dielectric loss at high frequency is an indication of the fact that the sample possesses superior optical quality with fewer defects and this parameter is of vital importance for NLO materials. Fig. 8 shows that the dielectric loss is very low at higher frequencies, indicating that the grown crystal contains the lowest number of defects $[17,18]$. The nature of dielectric constant variations with frequency and temperature indicates the type of mechanisms involved. High frequencies are associated with dipolar orientational, atomic, and electronic effects. The high value of the dielectric constant at low frequency and high temperature is due to the polarization of space charge, which depends on the purity and perfection of the crystal. Dipolar polarization, however, depends more predominantly on temperature compared to atomic or electronic polarization. However, the space charge polarization also contributes significantly to the low-frequency region due to electrode charge. Thermal energy activates more surface charge to induce the surface polarization apart to charge polarization. Consequently, the dielectric constant increases and the corresponding dielectric loss also varies. Due to surface charges, electrode polarization decreases with frequency, resulting in dielectric constant decrease. Just as the frequency increases, the polarization of the bound charge becomes more prevalent due to the lattice phonons influence compared to surface charge effects. Fig. 9 shows the variation of AC conductivity at various frequencies and temperatures. It is seen that the value of AC conductivity increases with an increase in the frequency. The various 
mechanisms for conduction include bond conduction, conduction in extended states, conduction in localized states near the band edge and the conduction in localized states near the Fermi level. The conduction observed in the present case can be attributed to the localized states near the Fermi level.

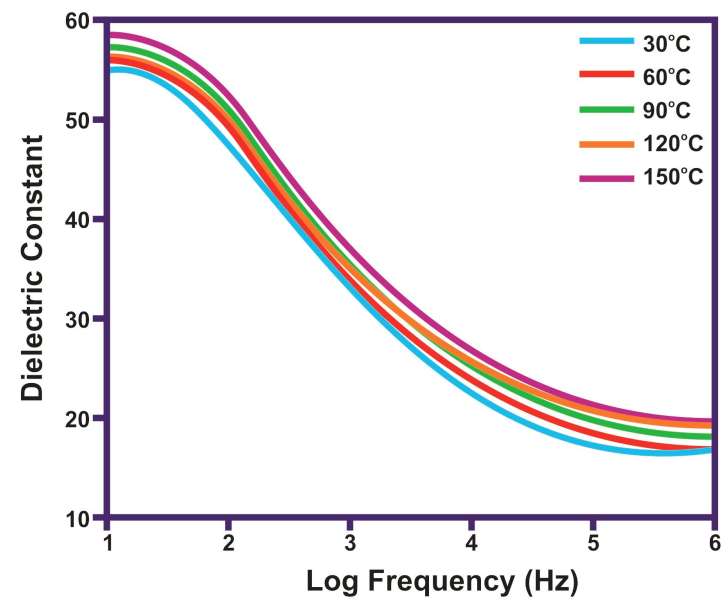

Fig. 7. Plots of dielectric constant vs. $\log$ frequency for $\mathrm{LCHF}$ at various temperatures.

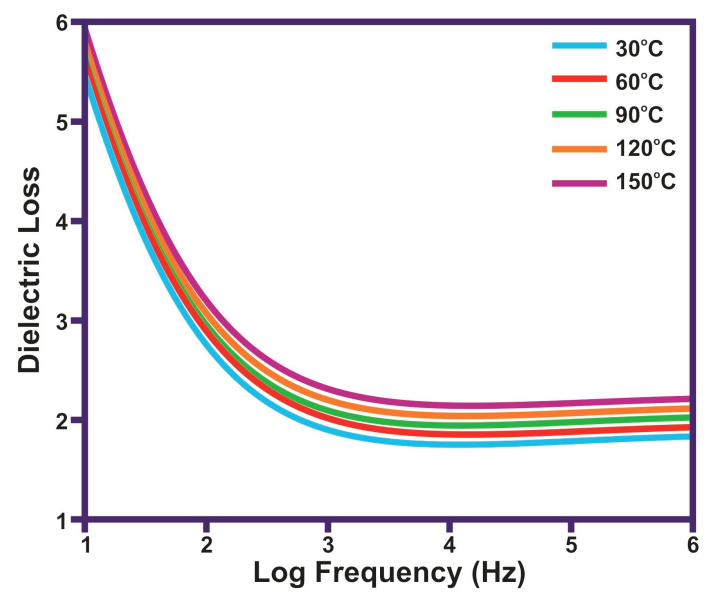

Fig. 8. Plots of dielectric loss vs. log frequency for $\mathrm{LCHF}$ at various temperatures.

\subsection{Vickers microhardness study}

Fig. 10 shows the variation of $\mathrm{H}_{\mathrm{V}}$ as a function of applied load, ranging from $10 \mathrm{~g}$ to $50 \mathrm{~g}$.

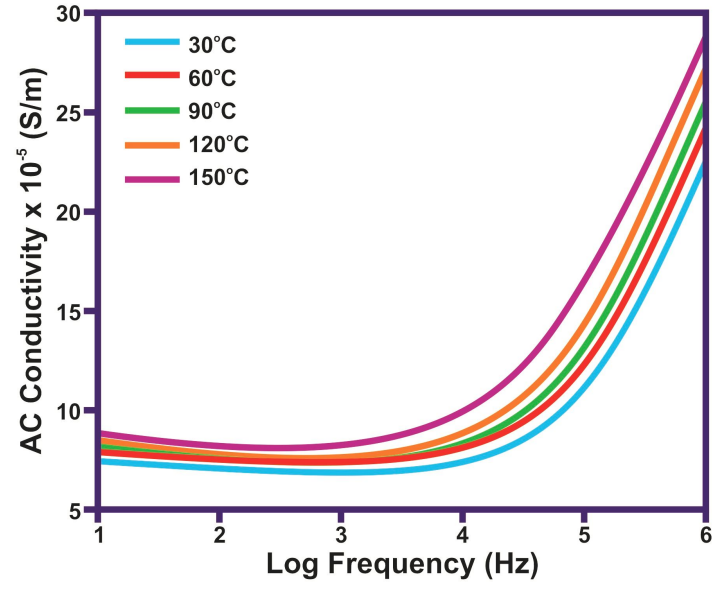

Fig. 9. Plots of AC conductivity vs. log frequency for LCHF at various temperatures.

It reveals that the hardness number increases as the load increases. This phenomenon is referred to as the effect of reverse indentation size (RISE). Dislocations are generated near the indentation site when the material is deformed by an indenter. The high stress required for homogeneous nucleation of dislocations in the dislocation free region [19] is attributed to the increase in hardness. RISE may be caused by relative nucleation predominance and dislocation multiplication. Essentially, the RISE phenomenon occurs in crystals that are readily subjected to plastic deformation [20]. The index $n$ of work hardening is obtained from the plot slope, $\log d$ vs. $\log P$ as shown in Fig. 11. The value of $n$ for LCHF was found to be 2.25. Low value of work hardening coefficient $\mathrm{n}$ illustrates fewer defects in the grown crystal. The value of $n$ is higher than 2 for soft materials and less than 2 for hard materials, according to Hanneman [21]. LCHF crystal, therefore, belongs to the category of soft materials.

\section{Conclusions}

The slow solvent evaporation technique in ambient environment was used to successfully synthesize transparent single crystals of L-cysteine hydrogen fluoride. Single crystal and powder XRD analyses estimated the lattice parameters that agreed well with the reported values. FT-IR investiations confirmed the presence of different functional 


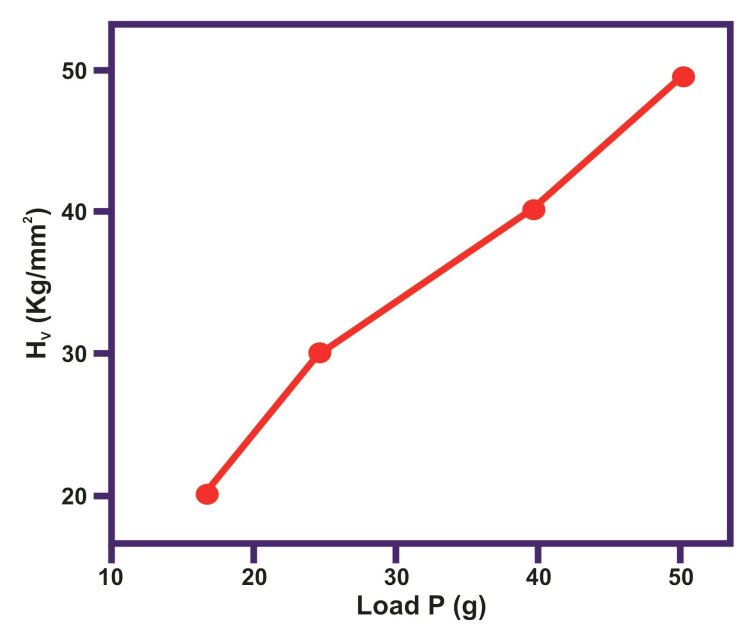

Fig. 10. Plot of hardness vs. load for LCHF.

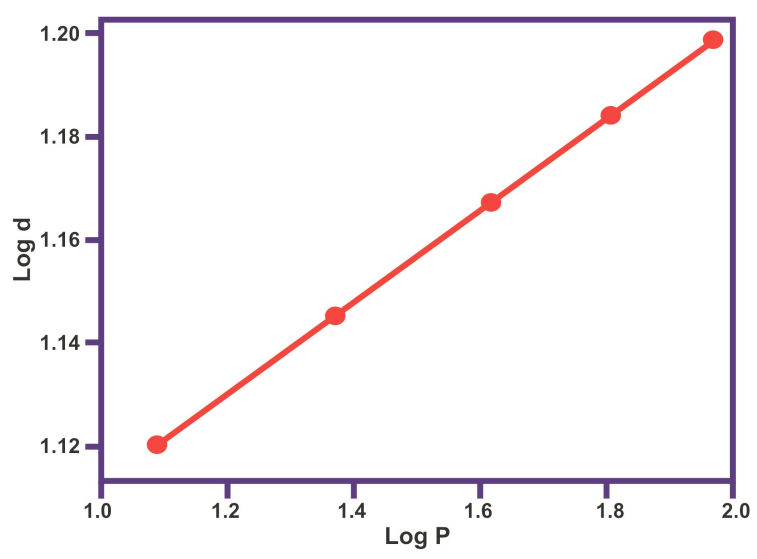

Fig. 11. Log d vs. $\log$ P for LCHF.

groups in the grown crystal. The presence of carboxylate ion, ammonium ion, and thiol group suggested that the obtained L-cysteine in LCHF existed in zwitterionic form. The UV-Vis-NIR transmission spectrum showed that the grown crystal was transparent with a lower edge at $228 \mathrm{~nm}$ in the visible region. The efficiency of SHG has been tested. Due to the addition of hydrogen fluoride, the thermal stability of LCHF increased by $13{ }^{\circ} \mathrm{C}$.
Dielectric measurements revealed that for electrooptic modulation, LCHF crystal showed a normal dielectric behavior. All these properties demonstrate that the title material can be a promising candidate for NLO device manufacturing.

\section{References}

[1] Suresh S., Optik, 127 (2016), 5613.

[2] Suresh S., Optik, 125 (2014), 2826.

[3] Suresh S., Optik, 125 (2014), 4547.

[4] Li L., Wang Z., Song X., Sun S., Spectrochim. Acta Part A, 72. (2009), 816.

[5] Naseema K., Rao V., Sujith K.V., Kalluraya B., Curr. Appl. Phys., 10 (2010), 1236.

[6] Singh S., Lal B., J. Crystal Growth, 312 (2010), 301.

[7] Bhat M.N., DharmapraKash S.M., J. Crystal Growth, 242 (2002), 245.

[8] Tlahuice-Flores A., J. Mol. Model., 19 (4) (2013), 1937.

[9] Tarakeshwar P., Manogara S., Spectrosc. Acta. Part A, 51. (1995), 925.

[10] Minko V.S., Ghazaryan W., Boldyreva E.V., Petrosyan A.M., Acta Crystallogr. C. Struct. Chem, 71 (8) (2015), 733.

[11] Petrosyan A.M., Terzyan S.S., Burbelo V.M., Sukiasyan R.P., Z. Naturforsch. Teil. A, 53 (1998), 528.

[12] Nithya N., Raman Mahalakshmi, Suresh S., Mater. Res., 18 (2015), 581.

[13] Kurtz S.K., Perry T.T., J. Appl. Phys., 39 (1968), 3798.

[14] Suresh S., J. Am. Chem. Sci., 3. (2013), 325.

[15] Koteeswari P., Mani P., Suresh S., J. Crystall. Proc. Tech., 2. (2012), 117.

[16] Suresh S., Mater. Phys. Mech., 14. (2012), 152.

[17] Suresh S., Arivuoli D., Lat. Am. J. Phys. Edu., 5 (2011), 721.

[18] Suresh S., Res. J. Chem. Sci., 2 (2012), 83.

[19] Suresh S., Priy A. M., Int. J. Rec. Adv. Phys., 3 (2014), 1.

[20] Sangwal K., Mater. Chem. Phys., 63 (2000), 145.

[21] Hanneman M., Metall. Manchu, 23 (1941), 135.

Received 2016-05-14 Accepted 2019-04-23 